\title{
Absceso retrofaríngeo por fístula congénita del seno piriforme en una adolescente
}

\author{
Retropharyngeal abscess due to a congenital pyriform sinus fistula in a teenager
}

Dra. Giselle Cuestas ${ }^{a}$, Dra. Verónica Rodrígueza , Dr. Patricio Bellia Munzón $n^{a}$ y Dr. Gastón Bellia Munzón ${ }^{b}$

\section{RESUMEN}

Las fístulas del seno piriforme son anomalías poco frecuentes de los arcos branquiales. La forma de presentación más común en los niños y los adolescentes es la tiroiditis aguda supurada y/o los abscesos cervicales laterales recurrentes. Sin embargo, las fístulas se pueden manifestar de forma atípica. La rareza de esta patología y la presentación clínica atípica pueden demorar el diagnóstico, lo que aumenta el riesgo de infecciones recurrentes y complicaciones.

Se presenta el caso inusual de una adolescente de 13 años con absceso retrofaríngeo debido a una fístula congénita del seno piriforme, tratada de forma exitosa mediante electrocauterización endoscópica.

Palabras clave: fistula, seno piriforme, absceso retrofaríngeo, endoscopía, electrocoagulación.

\begin{abstract}
Pyriform sinus fistulas are rare anomalies of the branchial arches. The most common form of presentation in children and adolescents is acute suppurative thyroiditis and / or recurrent lateral cervical abscesses. However, fistulas can manifest atypically. The rarity of this pathology and the atypical clinical presentation can delay the diagnosis increasing the risk of recurrent infections and complications.

We present the unusual case of a 13-year-old teenager with retropharyngeal abscess due to a congenital pyriform sinus fistula successfully treated by endoscopic electrocautery.

Key words: pyriform sinus, fistula, retropharyngeal abscess, endoscopy, electrocoagulation..
\end{abstract}

http: / / dx.doi.org/10.5546/ aap.2020.e81

Cómo citar: Cuestas G, Rodríguez V, Bellia Munzón P, Bellia Munzón G. Absceso retrofaríngeo por fístula congénita del seno piriforme en una adolescente. Arch Argent Pediatr 2020;118(1):e81-e84.

a. Sección de Endoscopía Respiratoria, División de Otorrinolaringología.

b. Servicio de Cirugía.

Hospital General de Niños "Dr. Pedro de Elizalde",

Ciudad Autónoma de Buenos Aires, Argentina.

Correspondencia:

Dra. Giselle Cuestas: giselle_cuestas@yahoo.com.ar

Financiamiento: Ninguno.

Conflicto de intereses: Ninguno que declarar.

Recibido: 22-5-2019

Aceptado: 22-8-2019

\section{INTRODUCCIÓN}

Las fístulas del seno piriforme (FSP) son anomalías poco frecuentes de los arcos branquiales, producto de la obliteración incompleta de la tercera o cuarta bolsa faríngea durante la séptima semana de gestación. ${ }^{1,2}$ Una teoría más reciente del origen de la malformación plantea que deriva del conducto timofaríngeo. ${ }^{2}$ Más del $95 \%$ se localizan en el lado izquierdo y se extienden desde el ápex del seno piriforme de la hipofaringe hasta la glándula tiroides o el espacio peritiroideo. $^{3}$

A pesar de estar presentes desde el nacimiento, las FSP se manifiestan, por lo general, hacia el final de la primera o en la segunda década de la vida. La forma de presentación más común en los niños y los adolescentes es la tiroiditis aguda supurada y / o los abscesos cervicales laterales recurrentes, principalmente, del lado izquierdo. ${ }^{3}$ Sin embargo, las FSP se pueden manifestar de forma atípica. Se han documentado unos pocos casos de abscesos retrofaríngeos y 1 caso de absceso submucoso esofágico secundarios a FSP. ${ }^{4-7}$

Como la enfermedad es poco frecuente y la presentación clínica es inespecífica, el diagnóstico, a menudo, se demora. Un alto índice de sospecha es de suma importancia para el diagnóstico preciso y el tratamiento oportuno de las FSP.

Se presenta a una paciente adolescente con absceso retrofaríngeo y tiroiditis aguda supurada debido a una FSP tratada mediante electrocauterización endoscópica.

\section{CASO CLÍNICO}

Paciente de sexo femenino de 13 años, que consultó por fiebre, rigidez cervical, odinofagia y disfagia a sólidos de 1 semana de evolución. No refirió historia de ingestión de ningún cuerpo extraño ni episodios previos similares. En el examen físico, presentó una masa dolorosa cervical anterolateral izquierda; el examen de fauces era normal.

El examen de laboratorio mostró la elevación de los indicadores de inflamación (glóbulos 
blancos: 17150/ml; valor normal -VN-: de 4500 a $11000 / \mathrm{ml}$; y proteína $C$ reactiva: $177 \mathrm{mg} / \mathrm{l}$; VN: $<8 \mathrm{mg} / \mathrm{l}$ ) y de los niveles de tirotrofina y tiroxina libre (T4L): la primera fue de $0,005 \mu \mathrm{UI} / \mathrm{ml}(\mathrm{VN}$ : de 0,4 a $4 \mu \mathrm{UI} / \mathrm{ml}$ ) y la segunda, de $2,48 \mathrm{ng} / \mathrm{dl}$ (VN: de 0,8 a $1,76 \mathrm{ng} / \mathrm{dl}$ ).

La ecografía cervical informó el aumento del tamaño del lóbulo tiroideo izquierdo con una colección en su interior compatible con absceso tiroideo y una colección adyacente a la glándula, sin poder precisar sus límites. La tomografía computada cervical con contraste endovenoso reveló la presencia de absceso retrofaríngeo y absceso paratiroideo izquierdo (Figura 1). Se realizó una nasofibrolaringoscopía con anestesia local y se observó un leve abombamiento de la pared posterior de la hipofaringe y un edema del seno piriforme izquierdo, lo que aumentó aún más la sospecha de FSP.

El tratamiento agudo inicial consistió en antibioterapia de amplio espectro $(2 \mathrm{~g} /$ día de ceftriaxona y $30 \mathrm{mg} / \mathrm{kg} /$ día de clindamicina por 9 días) y corticoides (8 $\mathrm{mg}$ de dexametasona cada 8 horas por 3 días) por vía endovenosa, seguidos por clindamicina oral $(600 \mathrm{mg} /$ dosis cada 8 horas por 10 días). La paciente evolucionó de forma satisfactoria.

Una vez superada la fase aguda, se realizó un examen endoscópico de la laringe e hipofaringe con óptica rígida $(5 \mathrm{~mm})$ bajo anestesia general. Se confirmó el orificio fistuloso en la fosa piriforme izquierda y se obliteró el trayecto de la fístula mediante cauterio monopolar (Figura 2). No hubo ninguna complicación asociada al procedimiento.

El egreso hospitalario se otorgó a las 6 horas de la observación. Se indicó dieta oral normal y analgesia según necesidad. A los 3 meses del tratamiento endoscópico, la paciente se encuentra asintomática, sin recidiva de la lesión. Los niveles hormonales de tirotrofina y T4L son normales.

\section{DISCUSIÓN}

El absceso retrofaríngeo es una infección profunda del cuello con potencial morbilidad y mortalidad. Se origina, por lo general, debido a una propagación de las infecciones de la vía aérea superior (faringitis, amigdalitis, adenoiditis, sinusitis, infecciones dentales o de las glándulas salivales) a los ganglios linfáticos localizados en ese espacio, que son prominentes en el niño pequeño $y$, habitualmente, sufren una involución a partir de los cuatro años. En general, en los pacientes de mayor edad, la infección de este espacio se produce a partir de un traumatismo penetrante o por la propagación directa de una infección desde un espacio adyacente, como en nuestro caso. ${ }^{8}$

La tiroiditis es rara en la edad pediátrica, debido a la alta resistencia a la infección de la glándula tiroides (por su alto contenido en iodo,

Figura 1. Tomografía computada del cuello. A, B y C. Absceso retrofaríngeo (flechas). A y B. Cortes sagitales. C. Corte coronal. D. Absceso paratiroideo izquierdo (flecha), corte coronal

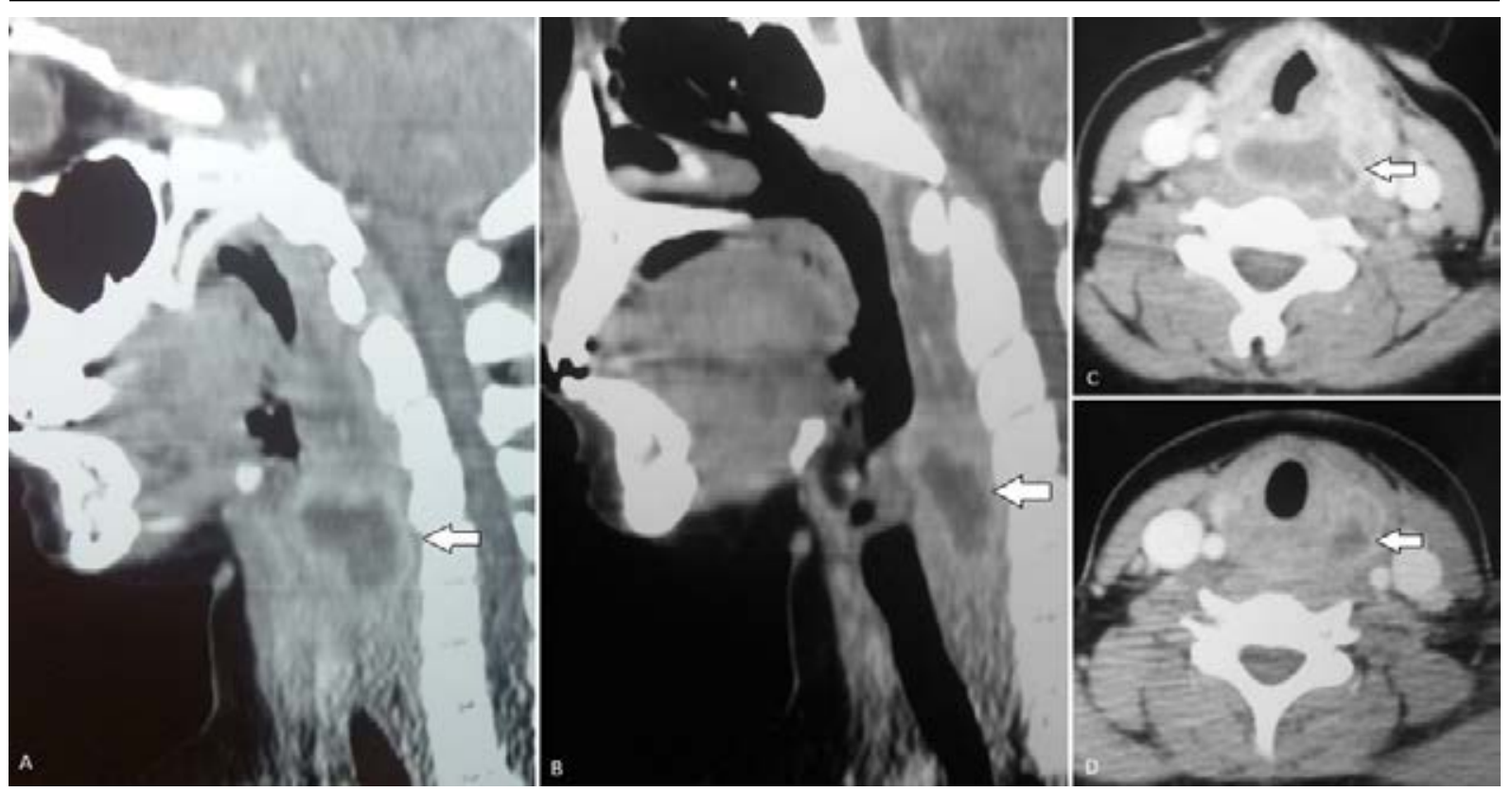


rica vascularización y cápsula fibrosa), por lo que se debe sospechar una FSP en todo niño o adolescente con tiroiditis. ${ }^{9}$ La progresión de la infección al espacio retrofaríngeo que presentó nuestra paciente era muy inusual. Se manifiesta, típicamente, con fiebre, disfagia y rigidez del cuello. La dificultad respiratoria y el estridor son menos frecuentes. ${ }^{10}$

La infección cervical es consecuencia de la contaminación del orificio interno de la FSP. Debido a la orientación de las capas de la fascia cervical profunda, es posible que una infección adyacente a la glándula tiroidea tenga acceso directo al espacio retrofaríngeo. ${ }^{6}$

La ecografía y la tomografía computada se realizan con frecuencia en la evaluación de la masa cervical en pediatría y son de gran utilidad para determinar el estado de la glándula tiroides, el sitio del absceso y la extensión de la lesión. Si aparece una inflamación tiroidea o un absceso adyacente, puede ser la primera pista hacia el diagnóstico de FSP. ${ }^{11}$
El esofagograma es útil para poner de manifiesto el trayecto fistuloso, pero pueden existir falsos negativos por la oclusión de este por un edema o secreciones. Por ello, la mayoría de los autores recomiendan realizar este estudio una vez resuelta la fase aguda. ${ }^{11}$

La endoscopía nasal con fibra óptica, realizada en el consultorio con anestesia tópica, puede revelar, ocasionalmente, la apertura de la fístula en la hipofaringe. El método diagnóstico de elección es la endoscopía rígida bajo anestesia general, que permite la visualización directa del orificio de la fístula y ofrece, además, la posibilidad de tratamiento inmediato $y$ potencialmente definitivo. ${ }^{3}$

El manejo clásico de la FSP consiste en antibioterapia de amplio espectro en la fase aguda, con o sin drenaje del absceso, y la posterior fistulectomía por vía cervical. ${ }^{6,7}$ Sin embargo, la identificación de la fístula puede ser dificultosa y existe el riesgo de lesionar estructuras adyacentes, como los nervios laríngeos superior y recurrente. ${ }^{3}$

Figura 2. Electrocauterización endoscópica de la fístula del seno piriforme. A. Se colocó un laringoscopio de suspensión, se expuso la fosa piriforme izquierda y se identificó el orificio de la fístula (flecha). La estrella indica la pared lateral de la laringe. B. Se introdujo por el orificio de la fístula una guía metálica envainada. C y D. Se obliteró el trayecto de la fístula (flecha) mediante cauterio monopolar (20w) de distal a proximal hasta observar que la mucosa cambiaba su coloración

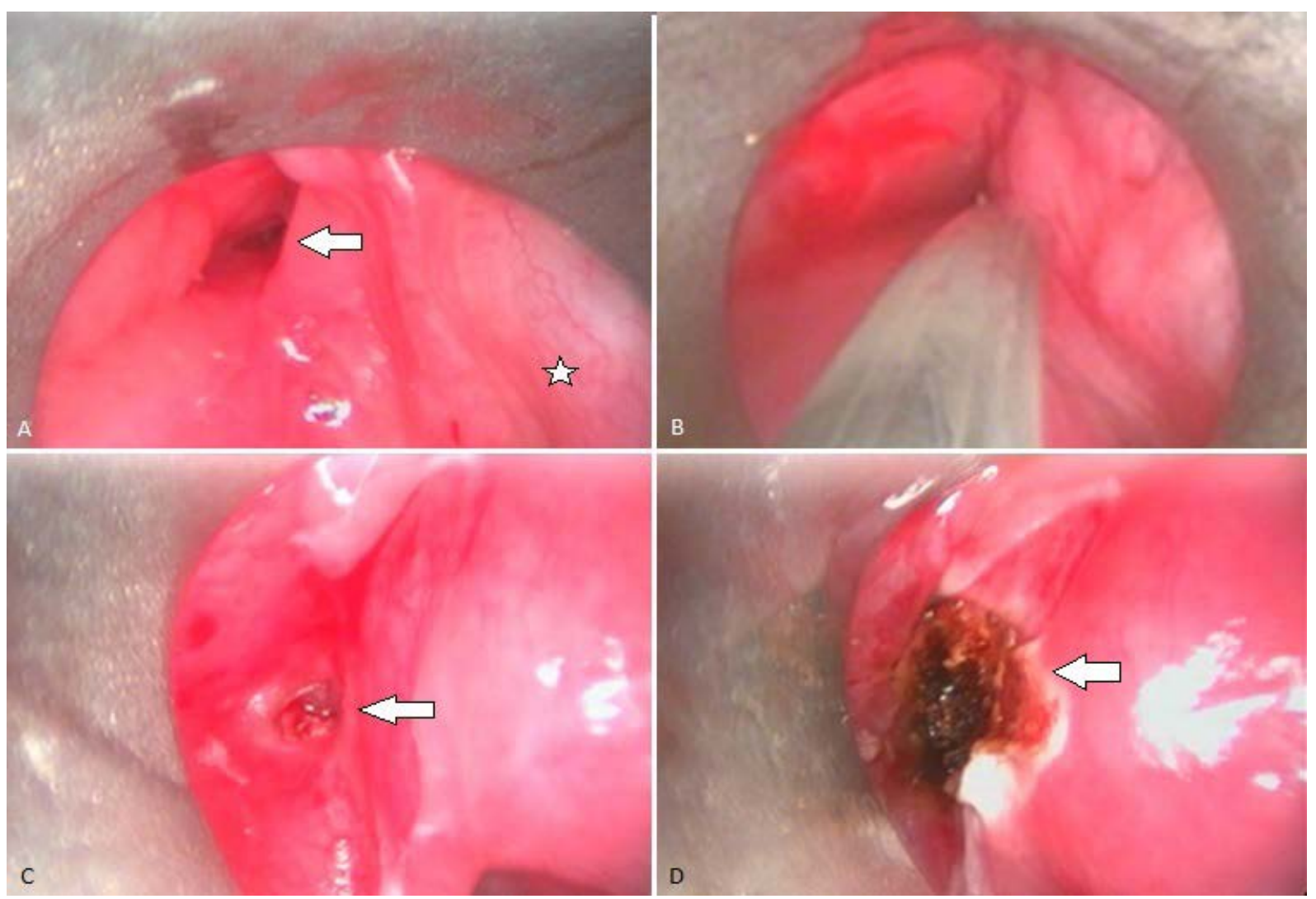


Como alternativa a la cirugía abierta, se han desarrollado técnicas menos invasivas y con menos riesgos de complicaciones que obliteran el trayecto de la fístula, como la electrocauterización endoscópica. ${ }^{3,12}$ El manejo endoscópico ha demostrado ser una alternativa segura y efectiva con una tasa de recurrencia comparable al manejo quirúrgico abierto (el $18 \%$ vs. el $15 \%$ ). ${ }^{13}$ Como la morbilidad es muy baja, se pueden realizar varios intentos endoscópicos de obliteración si se da la recurrencia de síntomas. En caso de fracasos reiterados del tratamiento conservador, se recomienda la cirugía. ${ }^{3,12}$

Es fundamental el seguimiento de los pacientes a largo plazo por la posibilidad de la recurrencia de la FSP, incluso años después de un procedimiento aparentemente exitoso. ${ }^{12} \mathrm{El}$ diagnóstico precoz y el tratamiento oportuno del trayecto fistuloso evitan el desarrollo de repetidas infecciones cervicales y complicaciones. Si bien es una rara forma de presentación, se debe considerar la FSP en el diagnóstico diferencial de absceso retrofaríngeo.

\section{REFERENCIAS}

1. Xiao X, Zheng S, Zheng J, Zhu L, et al. Endoscopic-assisted surgery for pyriform sinus fistula in children: Experience of 165 cases from a single institution. J Pediatr Surg. 2014; 49(4):618-21.

2. Cain RB, Kasznica P, Brundage WJ. Right-sided pyriform sinus fistula: A case report and review of the literature. Case Rep Otolaryngol. 2012; 2012:934968.
3. Cuestas G, Doormann F, Rodríguez V, Bellia Munzón $\mathrm{P}$, et al. Electrocauterización endoscópica de las fístulas congénitas del seno piriforme en pediatría. Serie de casos. Acta Otorrinolaringol Esp. 2017; 68(4):220-5.

4. Huang RY, Damrose EJ, Alavi S, Maceri DR, et al. Third branchial cleft anomaly presenting as a retropharyngeal abscess. Int J Pediatr Otorhinolaryngol. 2000; 54(2-3):167-72.

5. Erisen L, Coskun H, Parlak M, Ozmen A. Fourth branchial arch anomaly and pyriform sinus fistula as a rare cause of recurrent retropharyngeal abscess and thyroiditis in an adult. Otolaryngol Head Neck Surg. 2005; 133(4):644.

6. DeLozier HL, Sofferman RA. Pyriform sinus fistula: an unusual cause of recurrent retropharyngeal abscess and cellulitis. Ann Otol Rhinol Laryngol. 1986; 95(4 Pt 1):377-82.

7. Koyama S, Fujiwara K, Morisaki T, Fukuhara T, et al. Submucosal abscess of the esophagus caused by piriform sinus fistula treated with transoral video laryngoscopic surgery. ORLJOtorhinolaryngol RelatSpec. 2016; 78(5):252-8.

8. Acosta Navas B. Absceso retrofaríngeo. Rev Pediatr Aten Primaria. 2016; 18(69):e27-30.

9. GhaemiN, SayediJ, BagheriS. Acute suppurative thyroiditis with thyroid abscess: A case report and review of the literature. Iran J Otorhinolaryngol. 2014; 26(74):51-5.

10. Spini R, Arias E, Bordino L, Cohen D, et al. Abcesos profundos de cuello. Reporte de tres casos pediátricos. Arch Argent Pediatr. 2017; 115(5):e302-6.

11. Fernández Córdoba M, Gonzálvez Piñera J, Hernández Anselmi E, Ruíz Cano R. Tiroiditis agudas y abscesos cervicales como manifestaciones de las fístulas del seno piriforme. Cir Pediatr. 2009; 22(3):157-61.

12. Sun JY, Berg EE, McClay JE. Endoscopic cauterization of congenital pyriform fossa sinus tracts: an 18-year experience. JAMA Otolaryngol Head Neck Surg. 2014; 140(2):112-7.

13. Di Nardo G, Valentini V, Angeletti D, Frediani S, et al. Recurrent pyriform sinus fistula successfully treated by endoscopic Glubran 2 sealing: A rare case and literature review.SAGE Open Med Case Rep. 2016;4:2050313X16672151. 